\title{
MOVING SURFACE BOUNDARY LAYER CONTROL WITH APPLICATION TO HIGH PERFORMANCE AIRCRAFT
}

\author{
S. Saito, F. Mokhtarian \\ M.S.U.K. Fernando, V.J. Modi \\ Dept. of Mechanical Engineering \\ The University of British Columbia \\ Vancouver, B.C., Canada
}

\begin{abstract}
The concept of moving surface boundary-layer control, as applied to a Joukowsky airfoil, is investigated through a planned experimental program complemented by a flow visualization study. The moving surface was provided by rotating solid and scooped cylinders located at the leading edge, the trailing edge, or the upper leading edge of the airfoil. Results suggest that the concept is quite promising leading to a substantial increase in lift and a delay in stall. Depending on the performance desired, appropriate combinations of cylinder geometry, location and speed can be selected to obtain favourable results over a wide range of the angle of attack $(\alpha)$.

A flow visualization study using a water tunnel with food colouring as marker substantiates, rather spectacularly, effectiveness of the moving surface boundary layer control and qualitatively confirms complex character of the flow as predicted by the experimental data.
\end{abstract}

\section{KEY WORDS}

Moving surface boundary-layer control.

\section{INTRODUCTION}

Ever since the introduction of the boundarylayer concept by Prandtl, there has been a constant challenge faced by scientists and engineers to minimize its adverse effects and control it to advantage. Methods such as suction, blowing, vortex generators, turbulence promoters, etc., have been investigated at length and employed in practice with a varying degree of success. A vast body of literature accumulated over years has been reviewed rather

\footnotetext{
* Visiting Research Associate, Science University of Tokyo, Tokyo, Japan.

** Research Assistant

$\dagger$ Postdoctoral Fellow

$\ddagger$ Professor, Fellow ASME, BIS, CASI
}

effectively by several authors including Schlichting [1], Chang [2] and others. However the use of moving wall for boundary layer control has received relatively less attention.

Irrespective of the method used, the main objective of a control procedure is to prevent or at least delay the separation of boundary layer from the wall. A moving surface attempts to accomplish this in two ways:

- it retards the initial growth of the boundary layer by minimizing relative motion between the surface and the free stream;

- it injects momentum into the existing boundary layer.

The most practical application of moving wall for boundary-layer control was demonstrated by Favre [3]. Using an airfoil with upper surface formed by a belt moving over two rollers, he was able to delay separation until the angle of attack reached $55^{\circ}$ where the maximum lift coefficient of 3.5 was realized. Alvarez Calderon and Arnold [4] carried out tests on a rotating cylinder flap to evolve a high lift airfoil for STOL type aircraft. The system was flight tested on a single engine high wing research aircraft.

Of some interest is the North American Rockwell designed OV-10A aircraft which was flight tested by NASA's Ames Research Center [5- 7]. Cylinders located at the leading edges of the flaps are made to rotate at high speed with the flaps in lowered position. The main objective of the test program was to assess handling qualities of the propeller powered STOL type aircraft at higher lift coefficents. The aircraft was flown at speeds of $29-31 \mathrm{~m} / \mathrm{s}$, along approaches up to $-8^{\circ}$, which corresponded to a lift coefficient of about 4.3. In the pilot's opinion any further reductions in approach speed were limited by the lateral-directional stability and control characteristics.

In terms of trying to understand the phenomenon at the fundamental level Tennant's contribution to the field is significant. Tennant et al. [8] have conducted tests with a wedge shaped flap having a rotating cylinder as the leading edge. Flap deflection was limited to $15^{\circ}$ and the critical cylin- 
der velocity necessary to suppress separation was determined. Effects of increase in gap size (between the cylinder and the flap surface) were also assessed. No effort was made to observe the influence of an increase in the ratio of cylinder surface speed $\left(U_{c}\right)$ to the free stream velocity $(U)$ beyond 1.2. Subsequently Tennant et al. [9] have reported circulation control for a symmetrical airfoil with a rotating cylinder forming its trailing edge. For zero angle of attack, the lift coefficient of 1.2 was attained with $U_{c} / U=3$.

With this as background, the investigation reported here studies fluid dynamics of an airfoil with the moving surface boundary layer control using an extensive wind tunnel test program. Details of the models and test methodology are given in reference $[10]$ and hence purposely omitted here for conciseness.

\section{MODELS AND TEST PROCEDURES}

The wind tunnel model, a symmetrical Joukowsky airfoil of $15 \%$ maximum thickness to chord ratio, approximately $0.38 \mathrm{~m}$ along chord and $0.68 \mathrm{~m}$ long, spanned the tunnel test section, $0.91 \times 0.68 \times$ $2.6 \mathrm{~m}$, to create essentially two-dimensional conditions. To provide flexibility in locating the cylinder on the airfoil and permit testing of multicylinder configurations, a sectional design was adopted. The model consists of an aluminum skin wrapped around a metal frame with various sections of the surface removable, as required, to accomodate cylinders. A nose fill-in section replaced the leadingedge cylinder when it was not used as a rotating element.

The configurations tested (Fig. 1) include:

(a) the leading-edge cylinder;

(b) the leading-edge scooped cylinder;

(c) the upper-leading-edge cylinder;

(d) the upper-leading-edge scooped cylinder;

(e) the upper leading-edge cylinder and the trailingedge cylinder.

( $f$ ) the upper leading-edge scooped cylinder and the trailing-edge cylinder.

The model was provided with a total of 44 pressure taps distributed over the circumference to yield detailed information about the surface loading. Obviously, locating pressure taps on the surface of the cylinder typically rotating in the range of 2000 to $7000 \mathrm{rpm}$ would present considerable practical difficulty. The problem was resolved by measuring the pressure in the immediate vicinity of the cylinder rather than on the surface itself.

The tests were conducted at a Reynolds number of $4.62 \times 10^{4}$. The pressure plots were integrated in each case to obtain the lift coefficient. The lift was also measured independently using a strain gauge balance to assess two-dimensionality of the flow.

The wind tunnel test program was complemented by a flow visualization study using a water tunnel having a test-section of $20.32 \mathrm{~cm} \times 20.32 \mathrm{~cm} \times$ $2.44 \mathrm{~m}$ and capable of producing Reynolds number in the range $60-10,000$. A solution of food colouring in water (specific density $\approx 1$ ), injected upstream in a central vertical plane, provided a rather stable flow description at a water speed of $2 \mathrm{~cm} / \mathrm{sec}(\mathrm{Re} \approx 3000)$. The rate of injection was controlled with brass needle valves. Both, angle of attack and cylinder speeds were systematically changed and still photographs as well as a video movie taken.

\section{RESULTS AND DISCUSSION}

The relatively large angles of attack used in the experiments result in a considerable blockage of the wind tunnel test-section, from $21 \%$ at $\alpha=30^{\circ}$ to $30 \%$ at $\alpha=45^{\circ}$. The wall confinement leads to an increase in local wind speed, at the location of the model, thus resulting in an increase in the aerodynamic forces. Several approximate correction procedures have been reported in literature to account for this effect. However, these methods are mostly applicable to streamlined bodies with attached flow. A satisfactory blockage correction approach for a bluff body with separating shear layers is still not available.

With rotation of the cylinder(s), the problem is further complicated. As shown by the pressure data and confirmed by the flow visualization, the unsteady flow can be separating and reattaching over a large portion of the top surface. In absence of any reliable procedure to account for wall confinement effects in the present situation, the results are purposely presented in the uncorrected form.

Fig. 2 summarizes the effects of modification of the airfoil with the leading edge cylinder (Fig. 1 -ii) and the cylinder rotation. The base airfoil has a maximum lift coefficient of about 0.82 at an angle of attack of $10^{\circ}$. There is a penalty associated with the modified nose geometry as well as due to the gap, but even at the lowest rate of rotation of the cylinder $\left(U_{c} / U=1\right)$ the lift and stall characteristics are significantly improved. The airfoil exhibits a desirable flattening of the lift curve at stall. The maximum lift coefficient measured with $U_{c} / U=4$ was around 1.8 at $\alpha=28^{\circ}$ which is almost 2.2 times the lift coefficient of the base airfoil.

In order to assess the effect of the leading edge cylinder position, the configuration with a solid cylinder located at approximately $5 \%$ of the chord (upper leading edge cylinder, Fig. 1-iv) was tested in the range of $U_{c} / U$ upto 4 . The results showed the stall delayed to $48^{\circ}$ with $C_{L, \max }=1.95$ (Fig. $3)$. Note, at $\alpha=32^{\circ}$, the airfoil has stalled even with $U_{c} / U=2$. However, the higher speeds of the cylinder $\left(U_{c} / U \geq 3\right)$ are capable of keeping the upper surface flow attached. Even at a very high incidence of $\alpha=48^{\circ}$, it is remarkable that the cylinder rotation at $U_{c} / U=4$ is able to maintain partially attached flow over the upper surface. There was, as can be expected, an associated drag penalty due to the lack of any significant pressure recovery near the trailing edge.

Compared to the leading edge cylinder study reported earlier where for $U_{c} / U=4, C_{L, \max } \approx$ 1.75 and $\alpha_{\text {stall }} \approx 28^{\circ}$, now we have $C_{L, \text { max }} \approx 1.95$ with $\alpha_{\text {stall }} \approx 48^{\circ}$. This clearly suggests that location of the cylinder near the leading edge can significantly affect the airfoil performance. Thus there is room for a systematic study to arrive at an optimum location.

The use of a leading edge cylinder was shown to extend the lift curve, thus substantially increasing the maximum lift coefficient and delaying stall (Fig. 2). On the other hand, operating as a flap, the trailing edge cylinder rotation resulted in an improvement in the lift coefficient, at a given angle of attack, before stall. In order to combine these effects, the base configuration was modified to include both the leading and trailing edge cylinders (Fig. 1-iii). Although the leading edge cylinder rotation still appeared to be quite effective in improving the lift and stall characteristics of the base configuration, there was a substantial penalty as- 
sociated with the removal of the sharp trailing edge and replacing it with a cylinder.

It is, however, the combined effects of both the cylinders that is of interest here. The results, shown in Fig. 4, suggest some benefit due to rotation of the two cylinders together. Although the increase in $C_{L, \max }$ is rather modest (from 1.75 to 2.22 , around $27 \%$ ), and it fails to delay the stall further compared to the leading-edge-cylinder case (sharp trailing edge, Fig. 2), the lift coefficient at a given $\alpha$ is indeed increased significantly, as expected, due to the leftward shift of the plots. This, of course, is due to the added circulation contributed by the trailing edge cylinder. For example $C_{L}=0.7$ at $\alpha=8^{\circ}$ and $\left(U_{c} / U_{\text {l.e. }}=3\right.$ (Fig. 2), while for the same angle of attack and $\left(U_{c} / U\right)_{\text {l.e. }}=\left(U_{c} / U\right)_{\text {t.e. }}=3$ the corresponding $C_{L} \approx 1.38$, an increase of around $97 \%$. Similarly, $C_{L} \approx 1.43$ for $\alpha=16^{\circ}$ and $\left(U_{c} / U\right)_{l . e .}=4$. On the other hand, with both the cylinders rotating at $U_{c} / U=4$ the lift coefficient is around 2.15, a further gain of about $50 \%$. Note, this value of the maximum lift coefficient represents an increase of $160 \%$ with respect to the reference configuration $\left(C_{L, \max }\right.$ of about 2.22 vs. 0.85 , Fig. 4$)$.

Next, the attention was focussed on assessing the effect of the rotating cylinder geometry. To that end the rotating solid cylinder was replaced by a scooped geometry at the leading edge. The rotating air scoop appears to, in effect, slow down the flow over the lower surface and redirect more air over the upper surface. The partially separated flow with the solid cylinder is now reattached. At higher rates of rotation the leading edge scooped cylinder appears to the flow as effectively solid and there is no particular advantage in having the scoop. On the other hand, slower speeds of rotation appear to enhance the effect of the scoop. For example, even at the cylinder rotation speed as low as $U_{c} / U=0.125$ the airfoil remains unstalled at $\alpha=24^{\circ}$ (Fig. 5). This is in contrast to the separated flow at $\alpha=16^{\circ}$ with the solid cylinder rotating at $U_{c} / U=1$. A slight shift of the lift plots to the left suggests a small increase in circulation due to the scooped geometry. The main advantage of the scoop configuration is that it can provide the same beneficial effect of the normal rotating cylinder but at a much lower speed.

It may be of interest to point out that the experiments with the scooped cylinder were extremely difficult to conduct primarily due to the demanding balancing requirement. Even a small unbalance caused during machining of the $68 \mathrm{~cm}$ long cylinder was sufficient to cause vibration, particularly at higher speeds, which had to be controlled as the gap size is only $1 \mathrm{~mm}$. Thus the tolerances on machining and balancing were rather critical.

To achieve the same effect as the solid upper leading edge cylinder, but at a lower speed of rotation, the possibility of using a scooped cylinder was investigated. Fig. 6 shows the lift characteristics of the Joukowsky airfoil with the upper leading edge scooped cylinder. Note, the stall characteristics in general are superior to those of the solid cylinder. At a speed ratio as low as $U_{c} / U=0.1$, it can give a lift coefficient of 1.7 with a stall angle of $48^{\circ}$. At higher speeds, as expected, the performance approaches that of the solid cylinder. Compared to the previous results (Fig. 5), the scooped cylinder with $U_{c} / U=0.1$ performs almost as well as the solid cylinder spinning at $U_{c} / U=3$.

As pointedout before, the principle of operation of the scooped cylinder is somewhat differ- ent from that of the solid cylinder. Essentially, it works as a vortex generator at lower speed ratios as confirmed by the flow visualization pictures presented later. The vortex travelling over the upper surface reduces the pressure thus increasing the lift. As the speed of rotation increases, the vortex shedding frequency increases together with a corresponding reduction in the strength of the vortex finally approaching the solid cylinder case.

To further improve the performance, a trailing edge solid cylinder in conjunction with the upper leading edge scooped cylinder was investigated. The behaviour of the leading edge cylinder, while the trailing edge cylinder is stationary was also studied (Fig. 7). Several items of interest should be noted here. At the outset it is apparent the scooped cylinder improves the lift even at a very low rotation rates of 0.05 and $0.1\left(\alpha>8^{\circ}\right)$. In fact, for a given $\alpha$, the lift coefficients are higher than those obtained with $\left(U_{c} / U\right) l . e$. of 0.5 and 1 . Note, the stall is delayed to beyond $40^{\circ}$ and the $C_{L}$ of 1.24 for $U_{c} / U=0.05$ should be considered indeed remarkable (an increase of $51 \%$ over the base airfoil and $74 \%$ over the modified airfoil data), considering that even for $\left(U_{c} / U\right)$ l.e. $=2$, at $\alpha=40^{\circ}$ the $C_{L}=1.48$. For practical application, this is quite encouraging as the lower rate of rotation is preferred from vibration consideration. To better appreciate the effect of the trailing edge cylinder, the speed ratio $\left(U_{c} / U\right) t . e$. was varied while keeping the leading edge cylinder at $\left(U_{c} / U\right)$ l.e. $=0.1$. The results are shown in Fig. 8. In general, as seen earlier, the trailing edge cylinder flatens the stall and moves the lift curve to the left. Increasing the speed ratio progressively increases the lift at a given angle of attack.

Performance of the scooped cylinder at two different locations, operating at different speeds, with and without the trailing edge cylinder, is summarized in Fig. 9. Results of the standard Joukowsky airfoil (symmetrical, $15 \%$ thickness), with its $C_{L, \max }=0.85$ and $\alpha_{\text {stall }}=10^{\circ}$, serve as a reference.

The upper leading edge scooped cylinder at $U_{c} / U=3$ gives a $C_{L}$ of 2.2 and the stall is not evident even at $48^{\circ}$. When combined with the solid trailing edge cylinder at $U_{c} / U=3$, the lift coefficient at lower angles of attack increases without affecting the stall and giving a rather flat lift curve, which is desirable.

Upper leading edge scooped cylinder at $U_{c} / U=$ 0.1 shows rather interesting results. A lift coefficient of around 1.75 is achieved at $\alpha=48^{\circ}$. When combined with the trailing edge cylinder $\left(U_{c} / U=3\right)$, it gives a lift coefficient of around 1.2 at $8^{\circ}$, a $70 \%$ increase.

To better appreciate physical character of the flow in the presence of cylinder rotation, it would be useful to study the corresponding pressure distribution data. A typical set of results for a moderately high angle of attack of $\alpha=36^{\circ}$ are presented in Fig. 10, for the case of the upper leading edge scooped cylinder. At $U_{c} / U=0.5$, the flow on the upper surface is fully separated representing the total stall. As $U_{c} / U$ increases, the negative peak at the leading edge increases and the point of separation moves downstrcam, giving a smaller wake. At $U_{c} / U=4$, upper surface shows a fully attached flow. The corresponding $C_{L}$ vs. $\alpha$ plots were presented earlier in Fig. 6.

Effect of the angle of attack on the pressure distribution over an airfoil with the scooped cylinder at upper leading edge and rotating at $U_{c} / U=0.1$ is shown in Fig. 11 (for lift data see Fig. 6). 
At smaller angles the flow remains fully attached. As angle of attack increases the negative pressure peak at the upper leading edge increases leading to higher lift coefficients. At $\alpha=40^{\circ}$ the upper surface flow is fully separated but with a large negative pressure due to the vortices shed by the scooped cylinder, which in turn, leads to a large lift coefficient. However, since the flow is fully separated the corresponding drag coefficient is expected to be also quite high. This would result in a relatively smaller $C_{L} / C_{D}$ as shown in Fig. 12 . Note, $U_{c} / U=3$ represents aerodynamically better configuration giving higher $C_{L} / C_{D}$ values even at higher angles.

Thus, depending on the intended objective in terms of desired $C_{L, \max }$ and stall angle, one can select an appropriate configuration to initiate a preliminary design.

\section{Flow Visualization}

The flow visualization study showed, rather dramatically, effectiveness of this form of boundary layer control. Presented in Fig. 13 are a few of the typical pictures taken for an airfoil, at $\alpha=30^{\circ}$, with the leading edge cylinder rotating at different speeds. At $U_{c} / U=1$ a large well defined wake is quite apparent (Fig. 13a), with large scale vortices sweeping away downstream. However, with the solid cylinder rotating at $U_{c} / U=5$ (Fig. $13 \mathrm{~b}$ ) the size of the wake is significantly reduced and the flow remains attached over most of the upper surface of the airfoil. Figure 13c shows the effect when the solid cylinder is replaced by a scooped cylinder. As can be expected, except for the series of tiny vortices forming the separation stream line, the essential features are quite similar. This implies that the rotating scoop approaches the solid cylinder at higher speeds.

Distinctive contribution of the scooped cylinder becomes apparent at a lower speed of rotation. As noticed earlier it can produce high lifts at $U_{c} / U$ as small as 0.05 . Figure 14 shows the flow patterns for two values of cylinder rotation rate for $\alpha=20^{\circ}$. At $U_{c} / U=0.1$ a large vortex moving downstream over the upper surface is visible. This leads to a larger negative pressure on the upper surface thus giving a higher lift. However, the size of the wake is not reduced as in the previous case (Fig. 13b) by the cylinder rotation, which implies that the drag force is relatively larger. This was precisely revealed by the $C_{L} / C_{D}$ plots in Fig. 12 . When $U_{c} / U=0.5$, the scooped cylinder produces smaller vortices which move away from the surface without contributing effectively to the lift as shown in Fig. 8.

\section{CONCLUSIONS}

The experimental investigation with a symmetrical Joukowsky airfoil using leading edge, upper leading edge and trailing edge rotating cylinders, solid and scooped, brings to light several interesting aerodynamic features which may prove useful in the design of high performance airplanes:

- In general, rotation of the leading edge cylinder results in increased suction over the nose. However, it is the propagation of the lower pressure downstream, that determines effectiveness of the rotation. This depends mainly on the geometry of the nose and smoothness of transition from the cylinder to the airfoil surface.
- The increased momentum injection into the boundary layer, with an increase in speed of rotation, delays the separation of flow from the upper surface (stall) resulting in a higher $C_{L, \max }$. The existence of a critical speed is also evident beyond which momentum injection through a moving surface appears to have relatively less effect.

- With the rotation of the leading edge cylinder the onset of flow separation occurs at relatively higher angles of attack. The upper surface flow remains attached upto a distance downstream of the leading edge at which point it separates, leading to a large separation bubble, with reattachment towards the trailing edge. The flow, therefore, is not completely scparated from the airfoil thus resulting in a flatter stall peak.

- The use of a leading edge cylinder extends the lift curve without substantially changing its slope thus considerably increasing the maximum lift coefficient and stall angle. The Joukowsky model showed an increase in $C_{L, \max }$ by around $125 \%$ and the stall delayed from $10^{\circ}$ to $28^{\circ}$ with the solid leading edge cylinder.

- In contrast to the leading edge cylinder, the use of a trailing-edge cylinder substantially increases the lift before stall. The rotating trailing edge cylinder acts like a flap shifting the $C_{L}$ vs. $\alpha$ plots to the left. A high rate of rotation of this cylinder results in a dramatic increase in suction, over the airfoil upper surface, thus giving a larger lift. Furthermore, it can be used in conjunction with the leading-edge cylinder resulting in impressive values of lift over the whole range of low to moderately high angles of incidence $\left(\alpha \leq 18^{\circ}\right)$.

- The configuration with a rotating cylinder on the upper side of the leading edge appears to be quite promising. The increase in $C_{L, \max }$ at $U_{c} / U=4$ by around $167 \%$ and the delay in stall from $10^{\circ}$ to $48^{\circ}$ are indeed impressive.

- The upper leading edge scooped cylinder can produce larger lift at smaller speed ratios. However the reduction in pressure drag is not very significant since a recovery of pressure is not apparent at lower speed ratios. At larger speed ratios performance of the scooped cylinder approaches that of solid cylinder.

- The flow visualization study substantiate measured features of the flow and effectiveness of this form of boundary layer control in a convincing fashion.

\section{REFERENCES}

[1] Schlichting, H., Boundary Layer Theory, McGraw Hill Book Company, 1968.

[2] Chang, P.K., Separation of Flow, Pergamon Press, 1970.

[3] Favre, A., “Contribution a l'Etude Experimentale des Mouvements Hydrodynamiques a Deux Dimensions," Thesis presented to the University of Paris, 1938.

[4] Alvarez Calderon, A., and Arnold, F.R., "A Study of the Aerodynamic Characteristics of a High Lift Device Based on Rotating Cylinder Flap," Stanford University Technical Report RCF-1, 1961.

[5] Cichy, D.R., Harris, J.W., and MacKay, J.K., "Flight Tests of a Rotating Cylinder Flap on a North American Rockwell YOV-10A Aircraft," NASA CR-2135, November 1972. 
[6] Weiberg, J.A., Giulianettij, D., Gambucci, B., and Innis, R.C., "Takeoff and Landing Performance and Noise Characteristics of a Deflected STOL Airplane with Interconnected Propellers and Rotating Cylinder Flaps," NASA TM X62, 320, December 1973.

[7] Cook, W.L., Mickey, D.M., and Quigley, H.G., "Aerodynamics of Jet Flap and Rotating Cylinder Flap STOL Concepts," AGARD Fluid Dynamics Panel on V/STOL Aerodynamics, Delft, Netherlands, April 1974, Paper No. 10.

[8] Johnson, W.S., Tennant, J.S., and Stamps, R.E. "Leading Edge Rotating Cylinder for Boundary Layer control on Lifting Surfaces," Journal of Hydrodynautics, Vol. 9, No. 2, April 1975, pp. 76-78.

[9] Tennant, J.S., Johnson, W.S., and Krothapalli, A., "Rotating Cylinder for Circulation Control on an Airfoil," Journal of Hydronautics, Vol. 11 , No. 3, July 1976, pp. 102-105.

[10] Mokhtarian, F., "Fluid Dynamics of Airfoils with Moving Surface Boundary Layer Control, Ph.D. Thesis, University of British Columbia, February 1988.
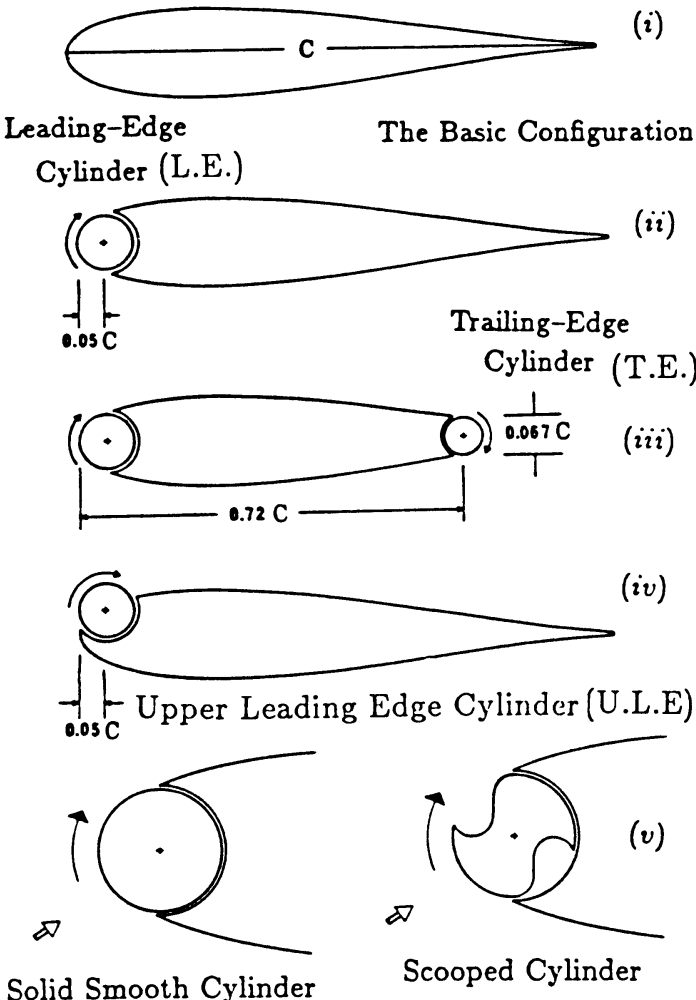

Fig. 1 Various rotating cylinder configurations used in the experimental program.

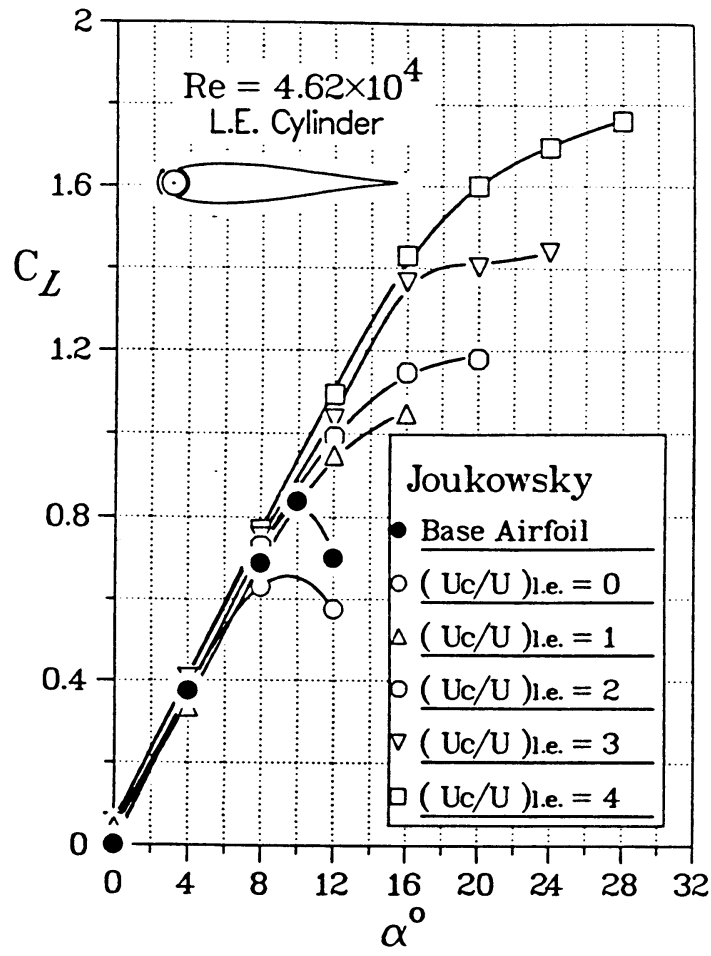

Fig. 2 Effect of the leading edge cylinder rotaion on the lift and stall characteristics.

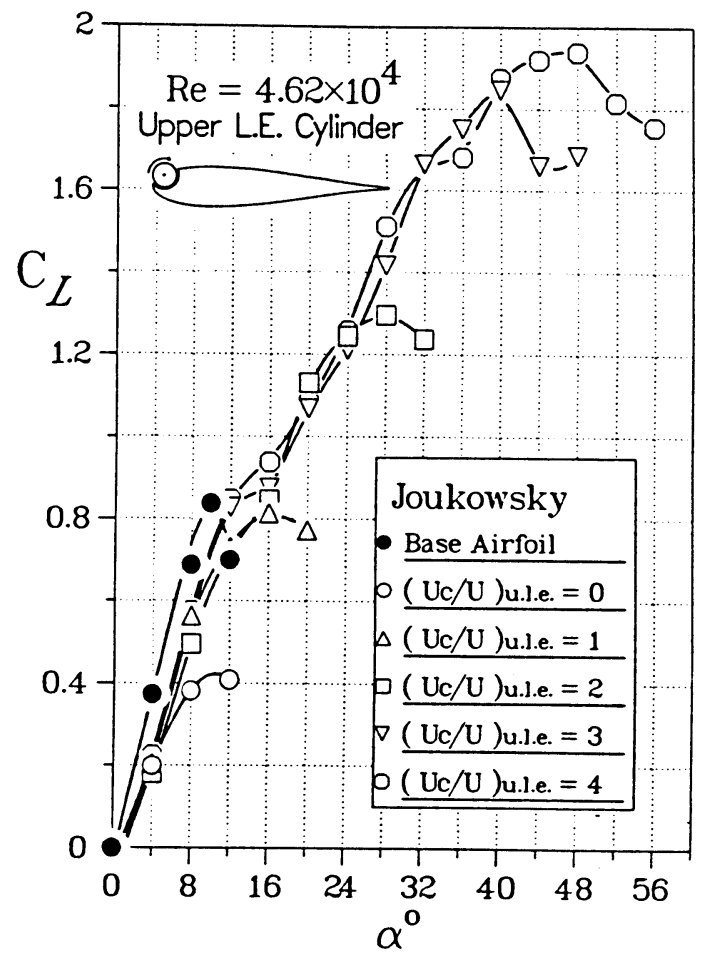

Fig. 3 Lift and stall characteristics of the Joukowsky airfoil as affected by the upper leading edge cylinder rotation. 


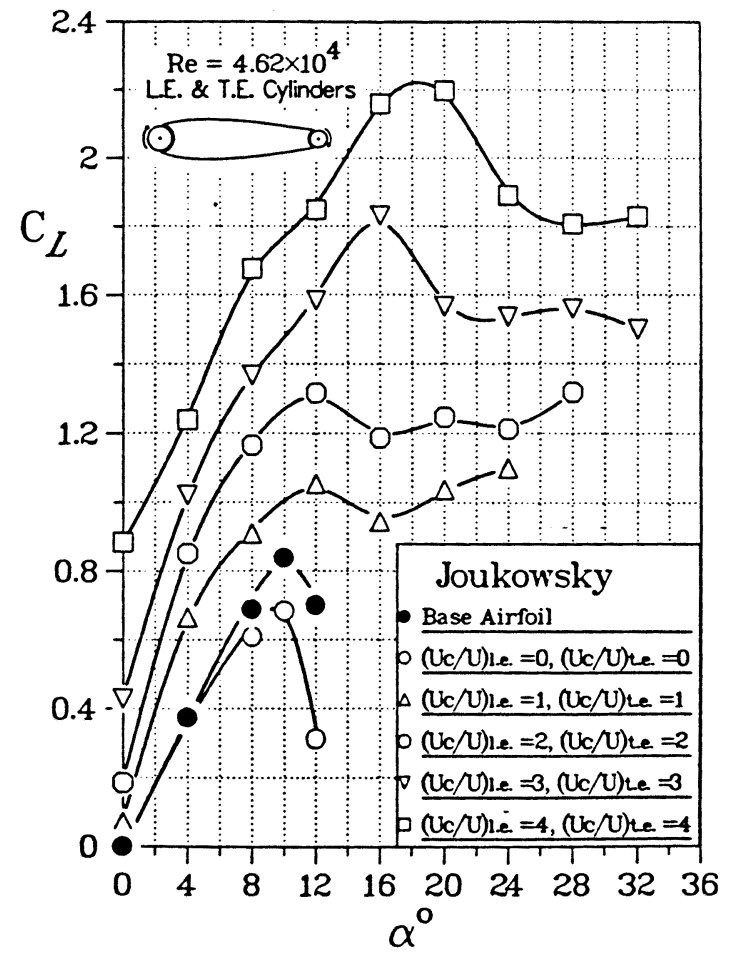

Fig. 4 Effect of the leading and trailing edge cylinder rotation on the lift and stall characteristics.

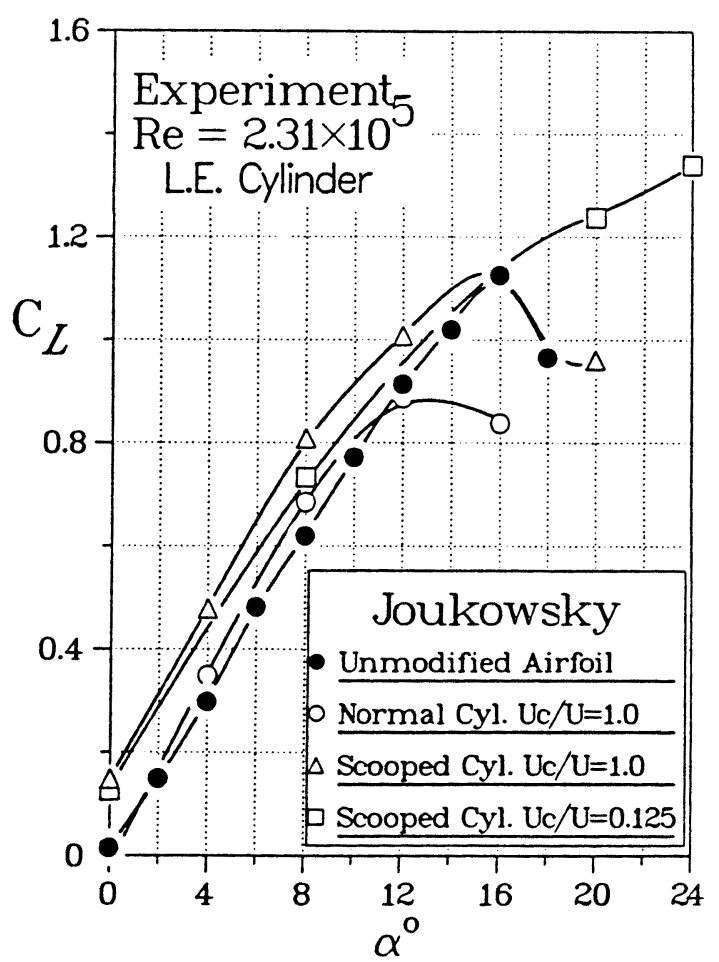

Fig. 5 Effect of the leading edge scooped cylinder rotaion on the lift and stall characteristics.

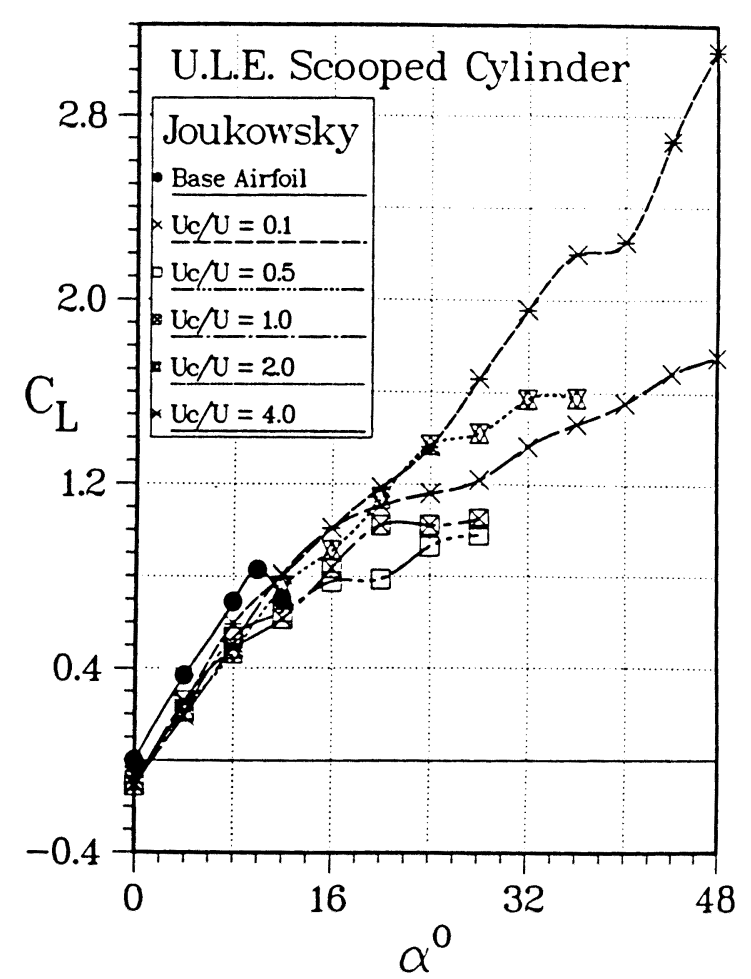

Fig. 6 Lift and stall characteristics of the Joukowsky airfoil as affected by the upper leading edge scooped cylinder rotation.

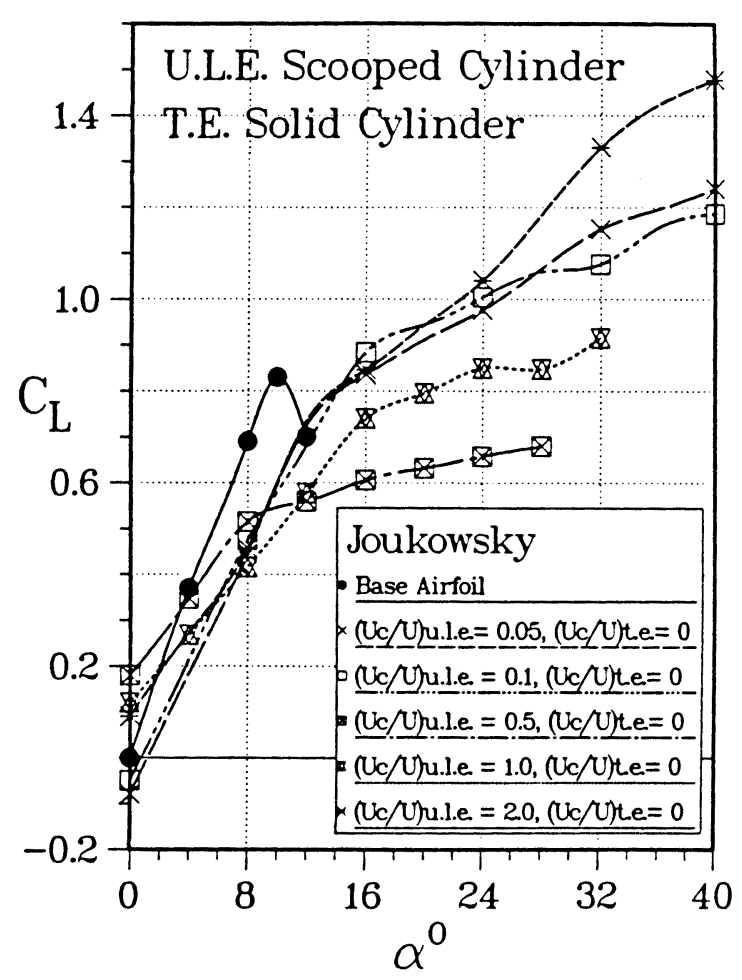

Fig. 7 Effect of the upper leading edge scooped cylinder rotation on the $C_{L}$ vs. $\alpha$ curve when the trailing edge cylinder is stationary. 


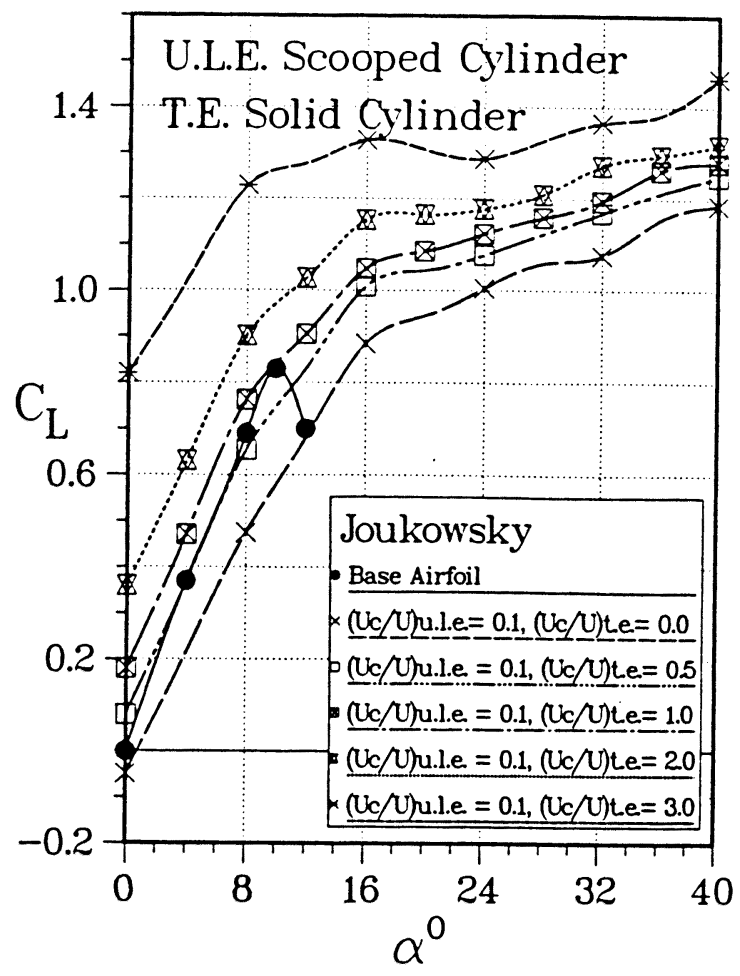

Fig. 8 Effect of the trailing edge cylinder rotation on the $C_{L}$ vs. $\alpha$ curve when the upper leading edge scooped cylinder is rotating at $U_{c} / U=0.1$.

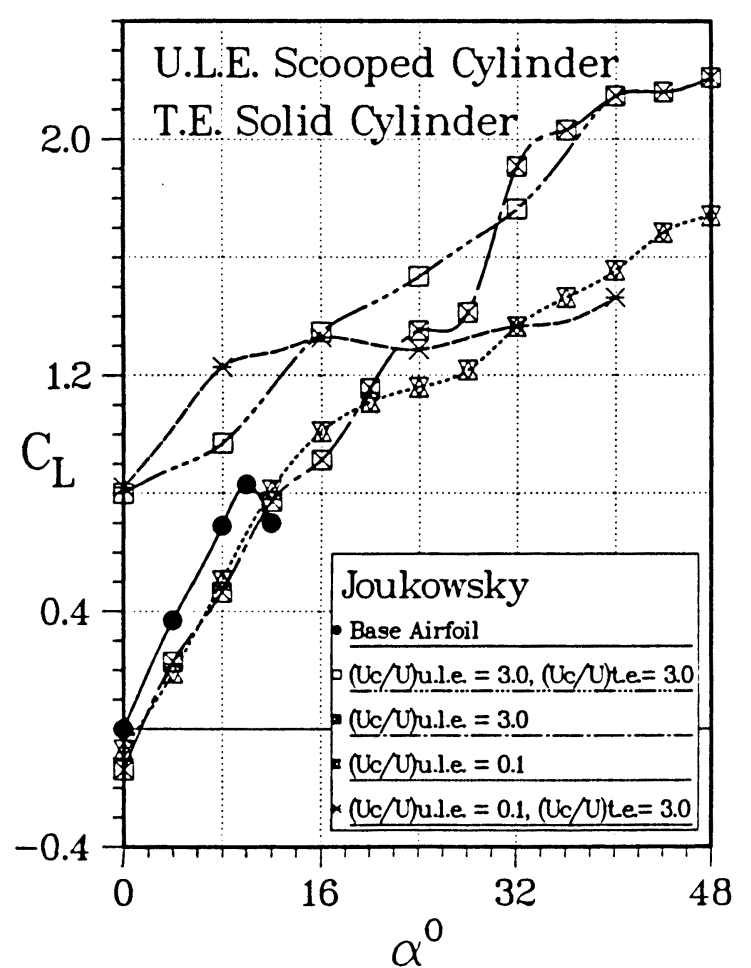

Fig. 9 Performance of the scooped cylinder at upper leading edge, operating at different speeds, with and without the trailing edge cylinder.

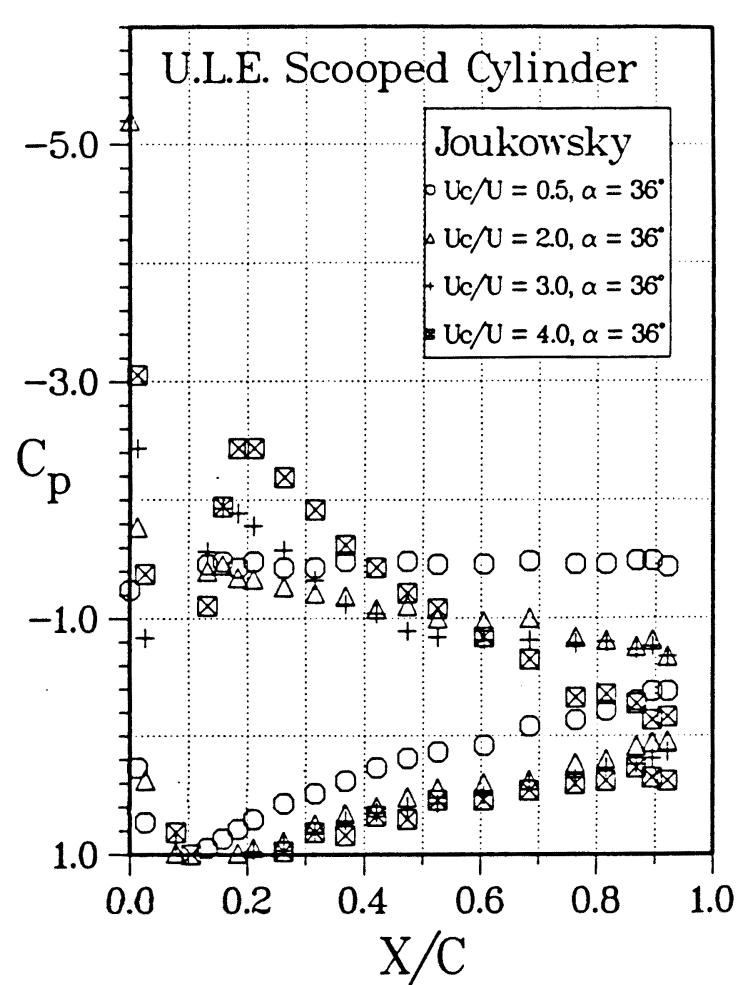

Fig. 10 Pressure distribution over the airfoil at $\alpha=36^{\circ}$ as affected by the scooped cylinder rotation rate.

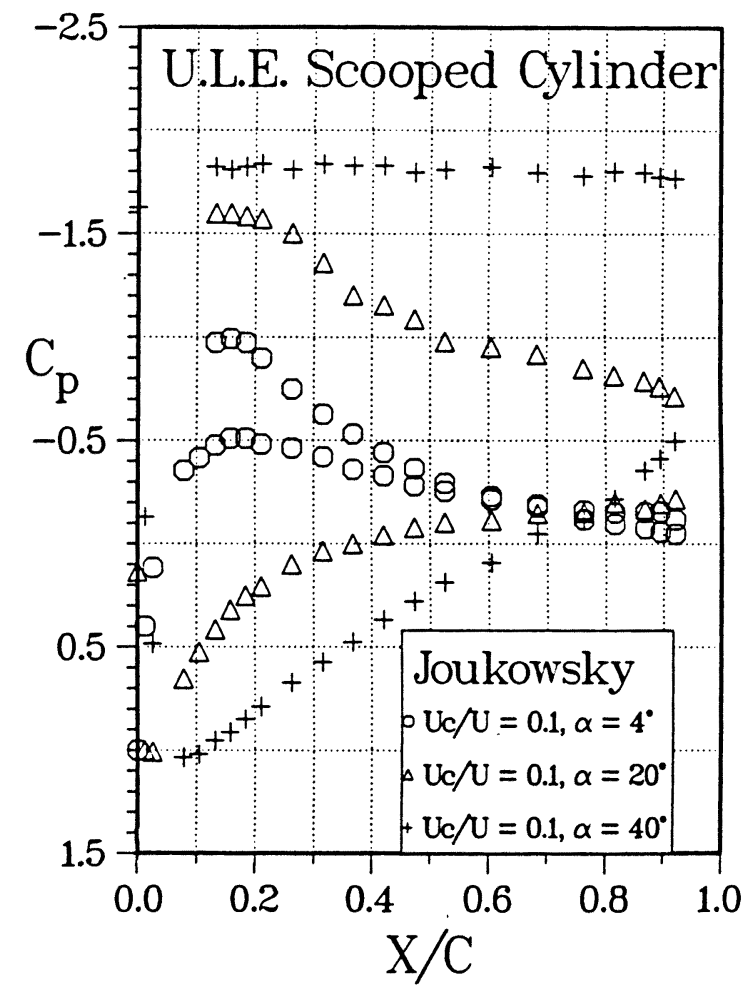

Fig. 11 Pressure distribution over the airfoil, with leading edge scooped cylinder rotating at $U_{c} / U=0.1$, as affected by the angle of attack. 


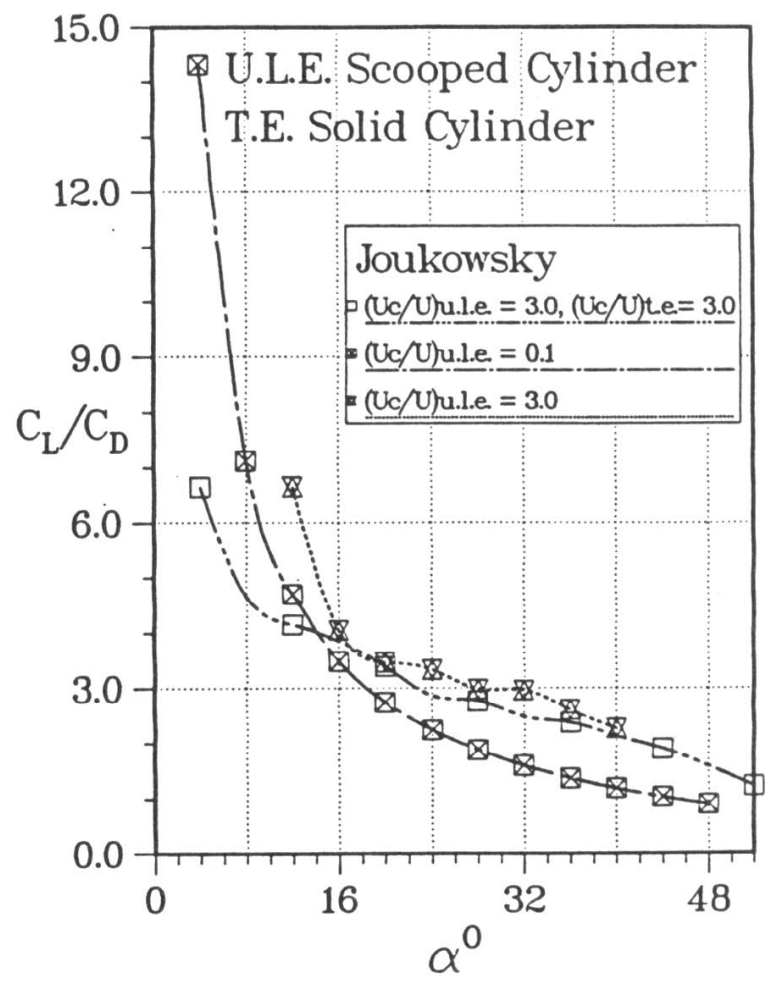

Fig. $12 C_{L} / C_{D}$ as affected by the angle of attack.
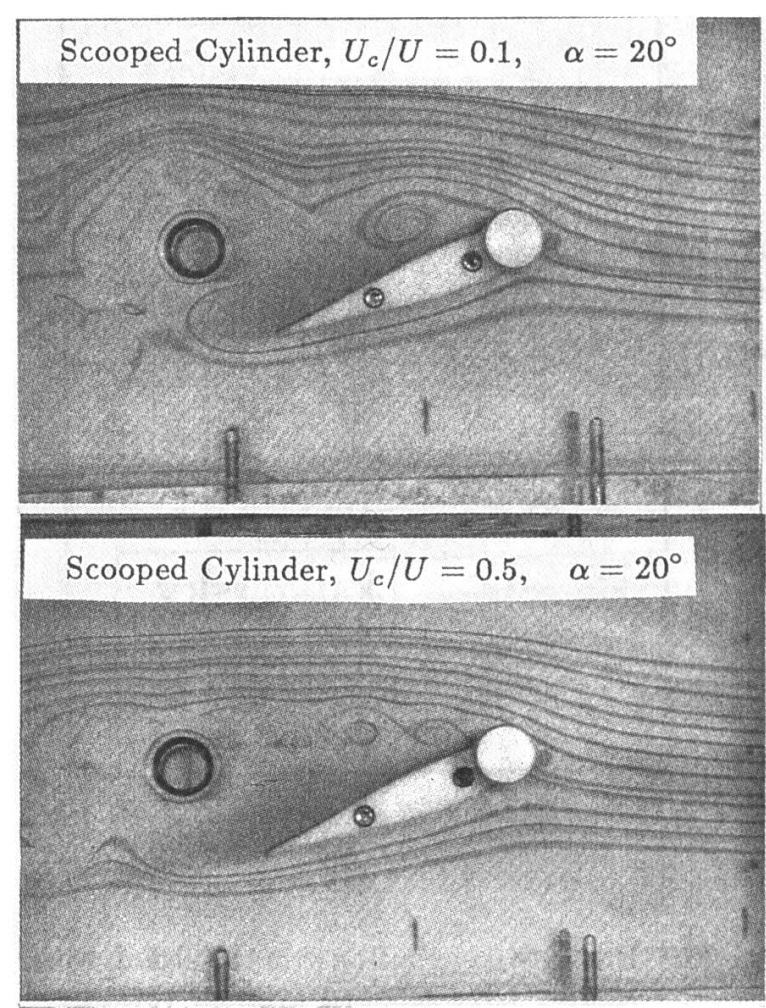

Fig. 14 Flow visualization pictures showing effectiveness of the scooped cylinder as a vortex generator at low speed of rotation.

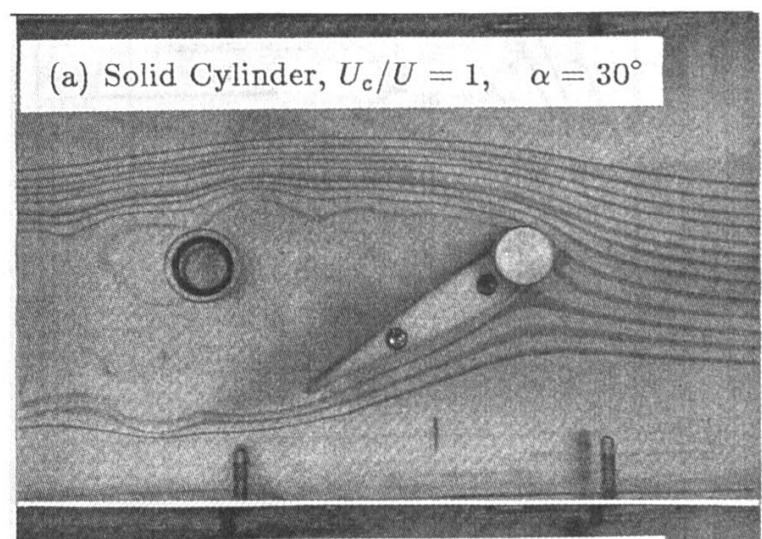

(b) Solid Cylinder, $U_{c} / U=5, \quad \alpha=30^{\circ}$

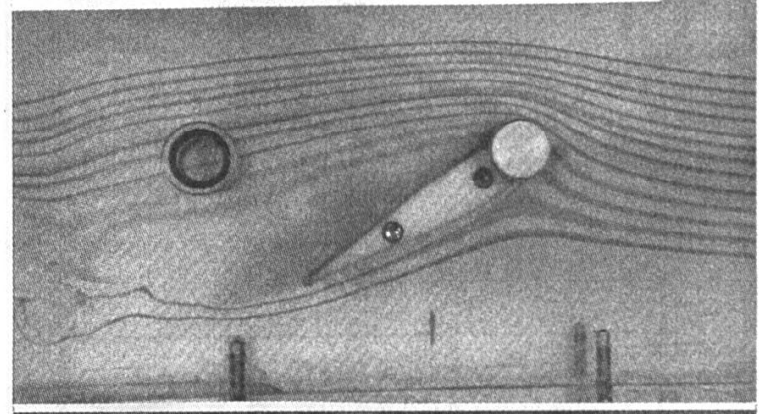

(c) Scooped Cylinder, $U_{c} / U=5, \quad \alpha=30^{\circ}$

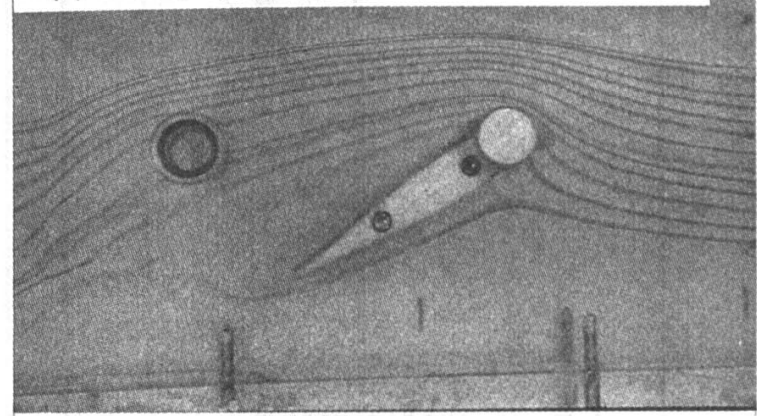

Fig. 13 Typical flow visualization pictures showing effectiveness of the moving surface boundary layer control. 